\title{
Regularity and Other Properties of Absolutely Continuous Invariant Measures for the Quadratic Family
}

\author{
Marek Rychlik ${ }^{1 \star}$ and Eugene Sorets ${ }^{2}$ \\ 1 The Institute for Advanced Study, School of Mathematics, Princeton, NJ 08540, USA \\ ${ }^{2}$ Courant Institute of Mathematical Sciences, New York, NY 10012, USA
}

Received June 1, 1989; in revised form April 16, 1992

\begin{abstract}
In the current paper we study in more detail some properties of the absolutely continuous invariant measures constructed in the course of the proof of Jakobson's Theorem. In particular, we show that the density of the invariant measure is continuous at Misiurewicz points. From this we deduce that the Lyapunov exponent is also continuous at these points (our considerations apply just to the parameters constructed in the proof of Jakobson's Theorem). Other properties, like the positivity of the Lyapunov exponent, uniqueness of the absolutely continuous invariant measure and exactness of the corresponding dynamical system, are also proved.
\end{abstract}

\section{Table of Contents}

1. Introduction. . . . . . . . . . . . . . . . . . . . . . 217

2. Mappings of Class BV. . . . . . . . . . . . . . . . . . . 219

3. Annihilating Segments. . . . . . . . . . . . . . . . 222

4. Non-Peripheral Spectrum of the P-F Operator . . . . . . . . . . 225

5. Continuity of the Invariant Density I. . . . . . . . . . . . . . 227

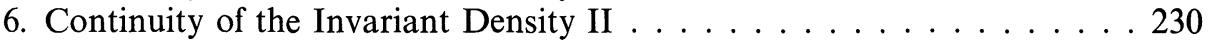

7. Continuity of the Lyapunov Exponent . . . . . . . . . . . . . 233

8. Appendix A: The Measure of the Central Segment . . . . . . . . 234

9. Appendix B: Positivity of the Lyapunov Exponent . . . . . . . . . . 235

References . . . . . . . . . . . . . . . . . . 236

\section{Introduction}

In [11] one of the authors (M.R.) presented a new proof of the following result of Jakobson [8]:

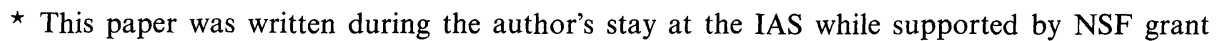
DMS-860 1978
} 
Theorem 1. Let $f_{\alpha}(x)=1-\alpha x^{2}, 0 \leqq \alpha \leqq 2$, be a one parameter family of mappings of the unit interval. There is a positive measure set of those $\alpha$ that $f_{\alpha}$ has an absolutely continuous invariant measure (abbreviation: a.c.i.m.).

In the course of the proof a set $A_{\infty}$ of parameters $\alpha$ was constructed such that for $\alpha \in A_{\infty}$ there is an a.c.i.m. for $f_{\alpha}$.

When the present work was started, our main objective was to show the continuity of the metric entropy, as $\alpha \rightarrow 2$. This naturally lead to studying continuity of the invariant measures and spectrum of the Perron-Frobenius operator for maps of class BV. In the present paper we also include a number of related results:

1. A direct, simple proof that for $\alpha \in A_{\infty}$ the Lyapunov exponent of $f_{\alpha}$ is positive; 2. A proof that the density of the a.c.i.m. is an $L^{p}$-function for $p \in[1,2)$.

Throughout the paper we use the results of [11] quite extensively. Also, in the part concerning BV class maps we refer the reader to [9] for an account of basic facts about these maps.

Let us recall that in [11] $A_{\infty}$ was constructed through a limiting process. First, for every nonnegative integer $n$ we constructed a family of segments $\mathscr{A}_{n}$. The segments of $\mathscr{A}_{n+1}$ were constructed from the segments of $\mathscr{A}_{n}$ by making deletions. The union of the segments of $\mathscr{A}_{n}$ was denoted by $A_{n}$. The set $A_{\infty}$ is simply the intersection of all $A_{n}$. Thus, for any $\alpha \in A_{\infty}$ a number of objects was constructed:

1. A descending sequence of segments $[-1,1]=I_{0} \supset I_{1} \supset I_{2} \ldots \supset\{0\}$; these segments depend on $\alpha$;

2. A sequence of integers $1=s_{0}<s_{1}<s_{2}<\ldots$ such that the mappings $T_{\alpha, k}=f_{\alpha}^{s_{k}} \mid I_{k} \backslash I_{k+1}, k=0,1,2, \ldots$ are uniformly expanding; the expansion constants $\rightarrow \infty$ exponentially with $k$ and uniformly in $\alpha$; the numbers $s_{0}, s_{1}, \ldots, s_{n}$ are the same for all $\alpha$ in the same segment $J \in \mathscr{A}_{n}$.

The mapping $T_{\alpha}$ was defined as $T_{\alpha, k}$ on the domain of $T_{\alpha, k}$ (i.e. on $M_{\alpha, k} \stackrel{\text { def }}{=} I_{k} \backslash I_{k+1}$. We proved that the mapping $T_{\alpha}$ belongs to class $\mathrm{BV}$, and therefore has an a.c.i.m. (cf. [9]). In the sequel we will show that the invariant measure for $T_{\alpha}$ is unique among the absolutely continuous ones. Let us denote it by $\bar{v}_{\alpha}$. Following the original work of Jakobson, we define the a.c.i.m. $v_{\alpha}$ for $f_{\alpha}$ via the following formula:

$$
v_{\alpha}=\sum_{n=0}^{\infty} \sum_{j=0}^{s_{n}-1}\left(f_{\alpha}^{j}\right) *\left(\bar{v}_{\alpha} \mid I_{k} \backslash I_{k+1}\right)
$$

This series defines an a.c.i.m. because the measure $\bar{v}_{\alpha}$ has a bounded density and the series

$$
\sum_{k=0}^{\infty} s_{k}\left|I_{k} \backslash I_{k+1}\right|
$$

converges (uniformly in $\alpha$ ). Up to a normalizing constant, this is the only a.c.i.m. for $f_{\alpha}$ (this fact will be evident in the sequel).

In Sect. 5 we show that the density of $\bar{v}_{\alpha}$ depends continuously on $\alpha$ in $L^{p}$-topology for any $p \in[1, \infty)$, as $\alpha$ converges to the so-called Misiurewicz points. Subsequently, (in Sect. 6) we prove an analogous statement for the density of $v_{\alpha}$ for $p \in[1,2)$. Our results are, of course, limited to the points in $A_{\infty}$. 
A parameter $\alpha$ is called a Misiurewicz point if the set $\left\{f_{\alpha}^{n}(0)\right\}_{n=1}^{\infty}$ is at a positive distance from the critical point 0 . It is not difficult to show that Misiurewicz points are dense in the set $A_{\infty}$. They are also Lebesgue density points of the set $A_{\infty}$.

Furthermore, we will show that the metric entropy which in this case equals the Lyapunov exponent of the dynamical system $\left(f_{\alpha}, v_{\alpha}\right)\left(\alpha \in A_{\infty}\right)$ is continuous at Misiurewicz points. We also show that the dynamical system $\left(f_{\alpha}, v_{\alpha}\right)$ is exact. From the results of Ledrappier [7] it follows that the natural extension of $\left(f_{\alpha}, v_{\alpha}\right)$ is Bernoulli. It shall be clear that the maps $T_{\alpha}$ have a uniformly exponential mixing rate for all $\alpha \in A_{\infty}$. An analogous statement for $f_{\alpha}$ was recently proven by L.-S. Young [12].

It is an open question whether for almost all $\alpha \in A_{\infty}$ the Lyapunov exponent along the orbit of 1 is positive. Again, the answer is well known to be positive for Misiurewicz points. Also, recently Carleson and Benedicks have shown that this is the case for the set of parameters constructed in their version of the proof of Jakobson's theorem. It is also known that for all $\alpha \in A_{\infty}$ :

$$
\left|\left(f_{\alpha}^{n}\right)^{\prime}(1)\right| \geqq \text { const. } \Lambda^{n^{\gamma}}
$$

for some $\Lambda>1$, all nonnegative integers $n$ and some positive $\gamma$. We expect that there is a nonempty set of measure 0 of these $\alpha \in A_{\infty}$ such that the Lyapunov exponent along the orbit of 1 is 0 .

Our technique relies upon the possibility to estimate the non-peripheral spectrum of the Perron-Frobenius operator of piecewise expanding mappings of class BV. Such estimates are directly connected with estimates of the rate of mixing and continuity of the entropy, as it was explained in [10].

\section{Mappings of Class BV}

The class of mappings considered in [9] will be called class BV. Thus, a mapping $T$ is of class BV if it maps $I \backslash S$ to $I$, where $S$ is a closed set of measure 0 ; $T$ is assumed to be differentiable and the function.

$$
g(x)= \begin{cases}\frac{1}{\left|T^{\prime}(x)\right|}, & \text { for } x \in I \backslash S \\ 0, & \text { for } x \in S\end{cases}
$$

satisfies $\operatorname{Var}(g)<\infty$ and $\|g\|_{\infty}<1$. Let $\xi$ be the measurable partition of $I$ into the components of $I \backslash S$. By enlarging $S$ we can achieve that $T$ is monotonic on the components of $I \backslash S$, the iteration $T^{n}: I \backslash S_{n} \rightarrow I$, where $S_{n}=\bigcup_{j=0}^{n-1} T^{-j}(S)$, is well defined and the partitions

$$
\xi^{n}=\bigvee_{j=0}^{n-1} T^{-j}(\xi)
$$

make sense. The function

$$
g_{n}=\left(g \circ T^{n-1}\right) \ldots(g \circ T) g
$$

plays the role of $g$ for the iteration. As it was shown in [9], $\operatorname{Var}\left(g_{n}\right)<\infty$, $\left\|g_{n}\right\|_{\infty} \leqq\|g\|_{\infty}^{n}<1$. So $T^{n}$ is also of class BV. 
The Perron-Frobenius operator for a BV map $T$ is an operator $P: L^{1}(m) \rightarrow L^{1}(m)$ defined as usual by the following formula:

$$
P \phi(x)=\sum_{y \in T^{-1}(x)} g(y) \phi(y) .
$$

$P$ can also be considered as an operator on the space BV of functions of bounded variation with the norm:

$$
\|\phi\|_{\mathrm{BV}}=\max \left(\|\phi\|_{L^{1}}, \operatorname{Var}(\phi)\right) .
$$

It will also be useful to denote by $\mathrm{BV}_{0}$ the space of those $\phi \in \mathrm{BV}$ which have zero integral. The following fundamental theorem was proved in [9]:

Theorem 2. There are constants $A, B, \kappa \in(0,1)$ such that for any $\phi \in \mathrm{BV}$ :

$$
\operatorname{Var}\left(P^{n} \phi\right) \leqq A \kappa^{n} \operatorname{Var}(\phi)+B\|\phi\|_{L^{1}}
$$

for $n=1,2, \ldots$.

From [9] it follows that the constants $A, B, \kappa$ can be obtained if a finite partition $\zeta$ and an integer $n$ are given, so that numbers $\lambda_{\zeta}$ and $D_{\zeta}$ (defined below) satisfy the following inequalities:

$$
\begin{gathered}
\lambda_{\zeta} \stackrel{\text { def }}{=}\left\|g_{n}\right\|_{\infty}+\max _{A \in \zeta} \operatorname{Var}_{A} g_{n}<1, \\
D_{\zeta} \stackrel{\text { def }}{=} \max _{A \in \zeta} \operatorname{Var}_{A}\left(g_{n}\right) /|A|<\infty .
\end{gathered}
$$

The above formulas are important to us because we will find the partition $\zeta$ independent of $\alpha$ such that both of the above constants are uniformly bounded for all $\alpha \in A_{\infty}$. As a consequence, the constants $A, B, \kappa$ can also be chosen uniformly with respect to $\alpha$.

Theorem 3. There is $\rho \in(0,1)$ and a constant $C$ such that for any segment $A \subset I$ and $N \in \mathbb{Z}^{+}$:

$$
\operatorname{Var}_{A}\left(g_{n}\right) \leqq C\left(\rho^{n}+|A|\right),
$$

where $C$ and $\rho$ do not depend on $\alpha$.

Proof. This proof is in principle the proof of the existence of the a.c.i.m. for $T$, which was hinted at in Remark 5.1 of [11]. Therefore, we will just sketch some of the steps, referring the reader to that paper.

Let $\pi$ be the partition of $I$ into segments of equal length $d$. Just for this proof, the meaning of $\mathscr{A}_{n}$ from [11] will be changed, to make the notation completely analogous to the proof of Theorem 5.1 from that paper. Thus, let $\mathscr{A}_{n}$ denote the partition $\xi^{n}$ restricted to $A$ and let $\mathscr{A}_{n}^{\prime}$ be the partition generated by the following inductive definition:

$$
\mathscr{A}_{n}^{\prime}=T^{-1}\left(\mathscr{A}_{n-1}^{\prime}\right) \vee(\xi \vee \pi)=\bar{\xi}^{n} \mid A,
$$

where $\bar{\xi}=\xi \vee \pi$. 
Let us introduce two sequences:

$$
\begin{gathered}
\gamma_{n}=\sum_{J \in \mathscr{A}_{n}^{\prime}} \sup _{J} \frac{1}{\left|\psi_{n}^{\prime}\right|}, \\
\eta_{n}=\sum_{J \in \mathscr{A}_{n}^{\prime}} \sup _{J}\left|\frac{\psi_{n}^{\prime \prime}}{\left(\psi_{n}^{\prime}\right)^{2}}\right||J|,
\end{gathered}
$$

where $\psi_{n}=T^{n}$. We will derive estimates of $\left(\gamma_{n+1}, \eta_{n+1}\right)$ in terms of $\left(\gamma_{n}, \eta_{n}\right)$ in a way analogous to the proof of Theorem 5 in [11].

For any $J \in \mathscr{A}_{n}^{\prime}, P \in \pi$ and $k \in \mathbb{Z}^{+}$we set $J_{k}(P)=T^{-1}(J) \cap M_{k} \cap P$. We notice that for any $J \in \mathscr{A}_{n+1}^{\prime}$ we have

$$
\sup _{J} \frac{1}{\left|\psi_{n+1}^{\prime}\right|} \leqq \sup \left|T_{(k)}^{\prime}\right|^{-1} \sup _{J_{k}(P)}\left|\psi_{n}^{\prime}\right|^{-1} .
$$

Let $k_{1} \in \mathbb{Z}_{+}$be such that (cf. formula (5.10) of [11])

$$
\sum_{k \geqq k_{1}} \sup \left|T_{(k)}^{\prime}\right|^{-1} \leqq \Lambda_{0}^{-1}
$$

Let us choose $d$ small enough, so that for every $P \in \pi$ one of the two cases holds: either 1) $P \subset I_{k_{1}}$ or 2) $P$ intersects not more than two of the sets $M_{k}$. We obtain:

$$
\sum_{k} \sup _{J_{k}(P)} \frac{1}{\left|\psi_{n+1}^{\prime}\right|} \leqq 2 \Lambda_{0}^{-1} \sup _{J_{k}(P)} \frac{1}{\left|\psi_{n}^{\prime}\right|}
$$

where $J(P)=\psi_{n}^{-1}(P) \cap J$. We notice that $\psi_{n}(J(P)) \neq P$ for at most two $P \in \pi$, namely the ones that satisfy $\left.{ }^{*}\right) P \cap \partial \psi_{n}(J) \neq \emptyset$. Summing up over these $P$ we obtain

$$
\sum_{k, P}^{*} \sup _{J_{k}(P)} \frac{1}{\left|\psi_{n+1}^{\prime}\right|} \leqq 4 \Lambda_{0}^{-1} \sup _{J} \frac{1}{\left|\psi_{n}^{\prime}\right|} .
$$

The other $P$ s satisfy **) $\psi_{n}\left(J_{k}(P)\right)=P$ and allow the estimate

$$
\sup _{J_{k}(P)} \frac{1}{\left|\psi_{n}^{\prime}\right|} \leqq \frac{1}{\left|\psi_{n}(J(P))\right|} \int_{\psi_{n}(J(P))} \frac{1}{\left|\psi_{n}^{\prime}\right|} \circ \psi_{n}^{-1}+\sup _{J(P)}\left|\frac{\psi_{n}^{\prime \prime}}{\left(\psi_{n}^{\prime}\right)^{2}}\right||J(P)| .
$$

So, we obtain

$$
\sum_{k, P}^{* *} \sup _{J_{k}(P)} \frac{1}{\left|\psi_{n+1}^{\prime}\right|} \leqq 2 \Lambda_{0}^{-1}\left(\frac{1}{d}|J|+\sup _{J}\left|\frac{\psi_{n}^{\prime \prime}}{\left(\psi_{n}^{\prime}\right)^{2}}\right||J|\right)
$$

and, finally

$$
\gamma_{n+1} \leqq 4 \Lambda_{0}^{-1} \gamma_{n}+2 \Lambda_{0}^{-1} \eta_{n}+\frac{2 \Lambda_{0}^{-1}}{d}|A| .
$$

Next we intend to estimate $\eta_{n+1}$. We have:

$$
\left|\frac{\psi_{n+1}^{\prime \prime}}{\left(\psi_{n+1}^{\prime}\right)^{2}}\right| \leqq \frac{1}{\left|T^{\prime}\right|} \circ \psi_{n}\left|\frac{\psi_{n}^{\prime \prime}}{\left(\psi_{n}^{\prime}\right)^{2}}\right|+\left|\frac{T^{\prime \prime}}{\left(T^{\prime}\right)^{2}}\right| \leqq \Delta_{x x}\left(T_{(k)}\right)+\Lambda_{0}^{-1}\left|\frac{\psi_{n}^{\prime \prime}}{\left(\psi_{n}^{\prime}\right)^{2}}\right| \text {. }
$$


Therefore, we can write:

$$
\eta_{n+1} \leqq \sum_{J, k, P} \Delta_{x x}\left(T_{(k)}\right)\left|J_{k}(P)\right|+\Lambda_{0}^{-1} \eta_{n}
$$

Because of the obvious inequality

$$
\left|J_{k}(P)\right| \leqq \sup _{J_{k}(P)} \frac{1}{\left|\psi_{n}^{\prime}\right|}\left|P \cap M_{k}\right|
$$

we obtain

$$
\eta_{n+1} \leqq r \gamma_{n}+\Lambda_{0}^{-1} \eta_{n}
$$

where

$$
r=\sup _{n} \max _{P \in \pi} \sum_{k} \Delta_{x x}\left(T_{(k)}\right)\left|P \cap M_{k}\right|
$$

Reasoning as in the proof of Theorem 5 of [11] we obtain the inequality:

$$
\mathbf{v}_{n+1} \leqq \mathbf{P v}_{n}+\mathbf{c} \quad(n=1,2,3, \ldots),
$$

where $\mathbf{v}_{n}=\left(\gamma_{n}, \eta_{n}\right) \in \mathbb{R}^{2}$ and $\mathbf{c} \in \mathbb{R}^{2}$ is a fixed vector satisfying the inequality:

$$
\|\mathbf{c}\| \leqq \text { const. }|A| \text {. }
$$

The matrix $\mathbf{P}$ is defined by the formula:

$$
\mathbf{P}=\left[\begin{array}{cc}
4 \Lambda_{0}^{-1} & 2 \Lambda_{0}^{-1} \\
r & \Lambda_{0}^{-1}
\end{array}\right],
$$

where

$$
r=\sum_{J, k, P} \Delta_{x x}\left(T_{(k)}\right)\left|J_{k}(P)\right|
$$

We need the spectral radius of $\mathbf{P}$ to be $<1$. This can be achieved by decreasing $d$, if $\Lambda_{0}>4$. Otherwise we use the same argument as in the proof of Theorem 5 in [11].

We also see from the definition and the estimates in [11] that $\mathbf{v}_{1}$ is uniformly bounded in $\alpha$.

As a corollary we obtain that if $\zeta$ is a sufficiently fine partition into segments then the numbers $\lambda_{\zeta}$ and $D_{\zeta}$ are uniformly bounded for all $\alpha \in A_{\infty}$.

\section{Annihilating Segments}

The purpose of this section is to introduce a condition which guarantees that the Perron-Frobenius operator of a $\mathrm{BV}$ map restricted to $\mathrm{BV}_{0}$ is a contraction. From the previous work on $\mathrm{BV}$ maps in [9] it is clear that this condition is equivalent to the weak mixing property for the resulting dynamical system, if the invariant measure is the unique a.c.i.m. However, we will need more concrete conditions which assure that the contraction is uniform for all maps $T_{\alpha}, \alpha \in A_{\infty}$.

Let $T$ be any BV map. 
Definition 1. Let $\varepsilon>0$. A segment $K_{0} \subset I$ is called an $\varepsilon$-annihilating segment if and for sufficiently large $l \in \mathbb{Z}_{+}$there are $C>0, \delta>0$ such that for any segment $K$ of length $>\varepsilon$ there is $B \in \xi^{l}$ and a subsegment $L \subset B$ such that:

1. $T^{l}(L)=K_{0}$ and $L \subset B \subset K$;

2. $|L| \geqq \delta$;

3. $\frac{\sup _{L} g_{l}}{\inf _{L} g_{l}} \leqq C$.

In the next section we will show that the existence of an $\varepsilon$-annihilating segment for sufficiently small $\varepsilon$ implies that the operator $P: \mathrm{BV}_{0} \rightarrow \mathrm{BV}_{0}$ is a contraction. Moreover, the contraction constant can be estimated in terms of the constants that have already been introduced.

In this section we will concentrate on the proof of the following result:

Theorem 4. For every $\varepsilon>0$ and for sufficiently small $\sigma>0$ the map $T=T_{2}$ (i.e. for $\alpha=2)$ the segment $K_{0}=[-\sigma, \sigma]$ is $\varepsilon$-annihilating.

Proof. Step 1. We change the coordinates, using the map $\Phi: x \mapsto \frac{2}{\pi} \arcsin (x)$. It is well known that the map $h=\Phi \circ f_{2} \circ \Phi^{-1}$ is actually the map $h: x \mapsto 1-2|x|$. For $\alpha=2$ the sequence of segments $I_{n}$ becomes $\left[-3^{-n}, 3^{-n}\right.$. Let us consider the sequence $I_{n}^{\prime}=\Phi^{-1}\left(I_{n}\right)=\left[-\sin \left(\frac{\pi}{2} 3^{-n}\right), \sin \left(\frac{\pi}{2} 3^{-n}\right)\right]$. Let $U$ be the map defined to be $h^{n+1}$, on $M_{n}^{\prime} \stackrel{\text { def }}{=} I_{n}^{\prime} \backslash I_{n+1}^{\prime}(n=0,1,2, \ldots)$. It is not difficult to see that

1. $U \mid M_{n}^{\prime}$ has slope $\pm 2^{n+1}$;

2. $\left|I_{n}^{\prime}\right| \sim$ const. $3^{-n}$ for large $n$;

3. $U=\Phi \circ T \circ \Phi^{-1}$.

Step 2. Let $\xi$ be the partition into the components of all the sets $M_{k}^{\prime}$. We shall prove that for sufficiently large $n$ there is $L \subset K_{0}, L \subset B \in \xi^{n}$, such that $K_{0}=U^{n}(L)$. Moreover, there is $m_{0}$ such that for every $m \geqq m_{0}$ there is a segment $L \subset M_{m}^{\prime}$ and $n \sim$ const. $m$ such that $U^{n}(L)=K_{0}$. Indeed, there is a sequence of preimages $K_{l}$ of $K_{0}$ under $h$ monotonically converging to -1 , all contained in $M_{0}^{\prime}$ (where $U=h$ ), of length $\sim K_{0} / 2^{l}$ and distant from -1 by $\sim 1 / 2^{l}$. The image $U\left(M_{m}^{\prime}\right)$ is a segment of length $\sim 2^{m}$ const. $3^{-m}$ and its distance from -1 is $\sim(2 / 3)^{m}$. It is clear now that for sufficiently large $l$ there is $m$ such that $U^{l+1}\left(M_{m}^{\prime}\right)=h^{l} U\left(M_{m}^{\prime}\right) \supset K_{0}$ provided $K_{0}=[-\sigma, \sigma]$ is short enough.

To prove the first of our claims it is sufficient to choose $l_{1}$ and $l_{2}$ so that $l_{1}+1$ and $l_{2}+1$ are relatively prime and the corresponding $I_{m_{j}} \subset K_{0}(j=1,2)$. Every sufficiently large $n$ admits a representation $n=p_{1}\left(l_{1}+1\right)+p_{2}\left(l_{2}+1\right)$, where $p_{1}, p_{2}$ are positive integers. Now using the representation $U^{n}=\left(U^{l_{1}+1}\right)^{p_{1}} \circ\left(U^{l_{2}+1}\right)^{p_{2}}$ we can easily find the desired segment $L$.

Step 3. Suppose that $K$ is a segment of length $>\varepsilon$. Let $K^{\prime}=\Phi(K)$. There is $\varepsilon_{1}>0$ independent of $K$ such that $\left|K^{\prime}\right|>\varepsilon_{1}$. We inductively define a sequence of segments $W_{n}$ by requiring that $W_{0} \subset K^{\prime},\left|W_{0}\right|>\varepsilon_{1} / 4$ and $\operatorname{dist}\left(W_{0}, 0\right) \geqq \varepsilon_{1} / 4$. For $n>0$ we define $W_{n}$ to be the longest of the segments $U\left(M_{k}^{\prime} \cap W_{n-1}\right)$ for $k=0,1, \ldots$ We claim that for some $n<$ const. $\log \left(1 / \varepsilon_{1}\right)$ the segment $W_{n}$ contains a component of $M_{m}^{\prime}$, where $m$ is sufficiently large and fixed. Indeed, if $W_{n}$ does not contain 
a component of $M_{m}^{\prime}$, then $W_{n}$ is either contained in $I \backslash I_{m+1}^{\prime}$ or in $I_{m}^{\prime}$. Let $m$ be a number so that

$$
\theta=\max \left(\sum_{k=1}^{m+1} \frac{1}{2^{k}}, \sum_{k=m}^{\infty} \frac{1}{2^{k}}\right)
$$

is strictly less than 1 . It is not difficult to prove by induction that for each $n$ we have $\left|W_{n+1}\right| \geqq \theta^{-1}\left|W_{n}\right|$. Indeed, suppose that, on the contrary, for each $k$ the length of $U\left(M_{k}^{\prime} \cap W_{n}\right)$ has length $<\theta^{-1}\left|W_{n}\right|$. We can write the following sequence of inequalities:

$$
\left|M_{k}^{\prime} \cap W_{n}\right|<\frac{1}{2^{k+1}} \theta^{-1}\left|W_{n}\right| \quad(k=0,1, \ldots) .
$$

In the case when $W_{n} \subset I \backslash I_{m+1}^{\prime}$ we sum up these inequalities for $k=0,1, \ldots, m$ and get a contradicion $\left|W_{n}\right|<\left|W_{n}\right|$. In the case when $W_{n} \subset I_{m}$ we sum up these inequalities for $k=m, m+1, \ldots$ and get the same contradiction. The inductive proof has been completed.

The inequality $\left|W_{n+1}\right| \geqq \theta^{-1}\left|W_{n}\right|$ persists as long as $W_{n}$ does not contain a component of $M_{m}^{\prime}$. Therefore, $n<$ const. $\log \left(1 / \varepsilon_{1}\right)$ and this proves the claim.

For sufficiently large $m$ the argument in Step 2 yields a subsegment $L^{\prime}$ and an iteration number $l$ such that $U^{l}\left(L^{\prime}\right)=K_{0}$. Let $L=\Phi\left(L^{\prime}\right)$. It is easy to see that this segment has the desired properties. Bounded distortion (condition 3 of Definition 1) follows from the fact that none of the segments $W_{n}$ can be too close to 0 and the fact that $T_{(k)}$ has bounded distortion (uniformly with respect to $\alpha$ ).

\section{Remark 1 .}

1. It is clear from the construction and the properties of $T_{(k)}$ that $K_{0}$ is an $\varepsilon$-annihilating segment for all $\alpha \in A_{\infty} \cap[2-\omega, 2]$ for sufficiently small $\omega$. Moreover, for fixed $\varepsilon$ and sufficiently large $l$ the choice of the constants $\delta$ and $C$ can be uniform for all parameters from $[2-\omega, 2]$.

2. The density of $v_{\alpha}$ is bounded away from 0 on $\left[f_{\alpha}(1), 1\right]=[1-\alpha, 1]$. To see that, let $K$ be an $\varepsilon$-annihilating segment of $T_{\alpha}$ and $S \subset K$ be an arbitrary non-empty subinterval. From uniqueness of a.c.i.m. for $T_{\alpha}$ it follows that

$$
\bar{v}_{\alpha}(S)=\sum_{n \geqq 0} m\left(T_{\alpha}^{-n} S\right) \geqq m\left(T_{\alpha}^{-l} S\right) \geqq \delta C^{-1} \frac{m(S)}{m(K)} \geqq \text { const. } m(S),
$$

where const. is positive and independent of $S$. It follows that the density of $\bar{v}_{\alpha}$ is bounded away from 0 on $K$. The $f_{\alpha}$ forward images of the annihilating segment cover $\left[f_{\alpha}(1), 1\right]$ by the proof of the last theorem.

3. The condition of exactness for $f$ (or any piecewise monotonic mappings of an interval preserving an absolutely continuous invariant measure) is equivalent to the following: For every non-empty interval $B$

$$
\lim _{n \rightarrow \infty} v\left(f^{n}(B)\right)=1
$$

It is clear that if $A=[-\sigma, \sigma]$ then $f^{n}(A)$ covers the set $[f(1), 1]$ (the support of $v$ ) in a finite number of steps (if $f=f_{\alpha}$ and $\alpha \in A_{\infty}$ ). Thus (34) is true for $A$. Now, the general statement follows from Theorem 4 and Definition 1. 
Indeed, let $B$ be an arbitrary segment of positive length and let $\sigma$ be such that the segment $A$ is $|B| / 2$-annihilating. Then for sufficiently large $l$ there is a segment $B^{\prime} \subset B$ such that $T^{l}\left(B^{\prime}\right)=A$ and $T^{l}$ is monotonic on $B^{\prime}$. Also, there exists $m$ such that $T^{l}\left(B^{\prime}\right)=f^{m}\left(B^{\prime}\right)$. Let $r$ be such that $f^{r}(A)=[f(1), 1]$. Thus, $f^{(m+r)}\left(B^{\prime}\right)=[f(1), 1]$, which means that $v\left(f^{(m+r)}(B)\right)=1$.

\section{Non-Peripheral Spectrum of the P-F Operator}

This section is crucial for the entire paper. Its goal is to estimate the spectral radius of the $\mathrm{P}-\mathrm{F}$ operator $P: \mathrm{BV}_{0} \rightarrow \mathrm{BV}_{0}$ of a $\mathrm{BV}$ class mapping $T$. It was known (cf. $[9,6])$ that this operator is a contraction, provided that the system possesses mixing property. However, the work prior to this paper does not provide an estimate for the contraction coefficient. It is apparent from the sequel that the need for such an estimate appears naturally in the problem of continuity of the metric entropy. A similar, simpler method was used in [10] to show continuity of the metric entropy for expanding mappings with respect to the unique absolutely continuous invariant measure.

For mappings of class BV the most important assumption we had to make is that the map has an annihilating segment (cf. Sect. 3). One can view the existence of an $\varepsilon$-annihilating segment as a way to quantitatively express the fact that the system has topological mixing. It is described quantitatively as a choice of constants $\delta, l$ and $C$ for an arbitrary $\varepsilon>0$.

Another ingredient we need in this section is the basic inequality:

$$
\operatorname{Var}\left(P^{n} \phi\right) \leqq A \kappa^{n} \operatorname{Var}(\phi)+B\|\phi\|_{L^{1}} .
$$

The way to compute the constants $A, B$ and $\kappa$ for any BV class map has been described in Sect. 2.

Theorem 5. The operator $P: \mathrm{BV}_{0} \rightarrow \mathrm{BV}_{0}$ is a contraction in a suitable norm equivalent to the norm of $\mathrm{BV}_{0}$. Moreover, there are constants $D<1$ and $l \in \mathbb{Z}$ such that $\left\|P^{l}\right\|_{\mathrm{Bv}_{0}} \leqq D$. These constants admit upper bounds depending on $A, B, C, \delta$ computed for some fixed $\varepsilon$, which will be described later.

Proof. Let us fix $l \in \mathbb{Z}_{+}$as in the annihilating segment condition for some sufficiently small $\varepsilon$ (described later) and $\gamma>0, R>0$. Let us consider a new norm on $\mathrm{BV}_{0}$ described by the following equation:

$$
\|\phi\|=\max \left(\operatorname{Var}(\phi), R\|\phi\|_{L^{1}}\right) .
$$

For any $\phi \in \mathrm{BV}_{0}$ let us consider the following two cases:

Case 1. $\operatorname{Var}(\phi) \geqq \gamma\|\phi\|_{L^{1}}$. In this case the basic inequality yields

$$
\begin{aligned}
& \operatorname{Var}\left(P^{l} \phi\right) \leqq\left(A \kappa^{l}+B / \gamma\right) \operatorname{Var}(\phi), \\
& R\left\|P^{l} \phi\right\|_{L^{1}} \leqq R\|\phi\|_{L^{1}} \leqq(R / \gamma) \operatorname{Var}(\phi) .
\end{aligned}
$$

So,

$$
\left\|P^{l} \phi\right\| \leqq \max \left(A \kappa^{l}+B / \gamma, R / \gamma\right)\|\phi\| .
$$

If $B / \gamma<1, R / \gamma<1$ and $l$ is sufficiently large, the norm of $\phi$ is contracted. 
Case 2. $\operatorname{Var}(\phi) \leqq \gamma\|\phi\|_{L^{1}}$. Let $K_{0}$ be an annihilating segment. We may assume that $\|\phi\|_{L^{1}}=1$ and obviously $\int \phi_{+} d m=\int \phi_{-} d m=1 / 2\left(\phi \in \mathrm{BV}_{0}\right)$.

Let $\Sigma_{1}=\left\{x \in I: \phi_{+}(x) \geqq 1 / 4\right\}$. We claim that $m\left(\Sigma_{1}\right) \geqq 1 /(4 \gamma)$. Indeed,

$$
\frac{1}{2}=\int \phi_{+} d m=\int_{\Sigma_{1}} \phi_{+} d m+\int_{I \backslash \Sigma_{1}} \phi_{+} d m \leqq \gamma m\left(\Sigma_{1}\right)+\frac{1}{4} m\left(I \backslash \Sigma_{1}\right) .
$$

Hence, we can write

$$
\gamma m\left(\Sigma_{1}\right) \geqq \frac{1}{2}-\frac{1}{4} m\left(I \backslash \Sigma_{1}\right) \geqq \frac{1}{4} .
$$

This implies that $m\left(\Sigma_{1}\right) \geqq 1 /(4 \gamma)$.

Our next claim is that there are at least $1 /(4 \gamma \varepsilon)$ disjoint segments of length $\varepsilon$ which contain a point of $\Sigma_{1}$. The proof is obvious.

We also observe that there are at most $8 \gamma$ disjoint segments such that on each of these segments the variation of $\phi$ exceeds $1 / 8$. Suppose that $\varepsilon<1 /\left(32 \gamma^{2}\right)$. This implies that $8 \gamma<1 /(4 \gamma \varepsilon)$ and there is a segment $K$ of length $\varepsilon$ such that $\inf _{K} \phi_{+} \geqq 1 / 8$. Consequently, there is a subsegment $L_{+} \subset K, L_{+} \subset B \in \xi^{l}$, such that:

1. $T^{l}\left(L_{+}\right)=K_{0}$;

2. $\sup _{L_{+}} g_{l} / \inf _{L_{+}} g_{l} \leqq C$;

3. $\inf _{L_{+}} \phi_{+} \geqq 1 / 8$;

4. $\left|L_{+}\right| \geqq \delta$.

In a similar way we define a segment $L_{-}$. It is not difficult to notice that $\left\|P^{l}\right\|_{L^{1}}<1$, due to the fact that the segments $L_{+}$and $L_{-}$meet after $l$ iterations and some part of the measure $\phi m$ will be "annihilated." A more precise estimate yields

$$
\left\|P^{l} \phi\right\|_{L^{1}} \leqq 1-\frac{1}{4} C^{-1} \delta \text {. }
$$

Indeed, we always have $\left|P^{l} \phi\right| \leqq P^{l}|\phi|$ and because of cancellations, on $K_{0}$ we have:

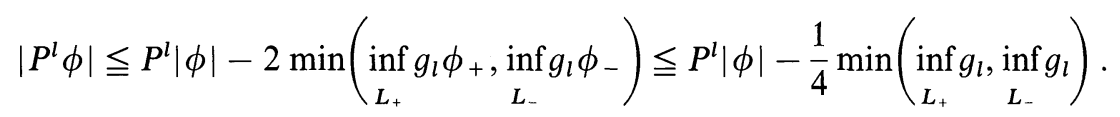

Integrating these inequalities we obtain:

$$
\left\|P^{l} \phi\right\|_{L^{1}} \leqq\|\phi\|_{L^{1}}-\frac{1}{4} m\left(K_{0}\right) \min \left(\inf _{L_{+}} g_{l}, \underset{L_{-}}{\inf } g_{l}\right),
$$

and since

$$
\inf _{L_{ \pm}} g_{l} \geqq C^{-1} \sup _{L_{ \pm}} g_{l} \geqq C^{-1} \frac{m\left(L_{ \pm}\right)}{m\left(K_{0}\right)} \geqq C^{-1} \frac{\delta}{m\left(K_{0}\right)},
$$

(41) follows. The basic ineqality yields

$$
\operatorname{Var}\left(P^{l} \phi\right) \leqq\left(A \kappa^{l} \gamma+B\right)\|\phi\|_{L^{1}} \leqq\left(\left(A \kappa^{l} \gamma+B\right) / R\right)\|\phi\|,
$$

and by (41) we get:

$$
\left\|P^{l} \phi\right\| \leqq \max \left(1-\frac{C^{-1} \delta}{4}, \frac{A \kappa^{l} \gamma+B}{R}\right)\|\phi\| .
$$


The conclusion for Cases 1 and 2 is that if $B / \gamma<1, R / \gamma<1, B / R<1$, and $l$ is sufficiently large then $P^{l}$ is a contraction with respect to the norm $\|\cdot\|$. All other claims follow easily, and the details are left to the reader.

\section{Continuity of the Invariant Density I}

Let us recall that $P_{\alpha}$ (the Perron-Frobenius operator of $T_{\alpha}$ ) is a bounded operator either in $L^{1}$-topology or BV-topology. But it is not true that $P_{\alpha}$ depends continuously on $\alpha$ in either topology. The density $\rho_{\alpha}$ of the a.c.i.m. in an eigenvector of $P_{\alpha}$ corresponding to the eigenvalue 1 . We are going to show that $\rho_{\alpha}$ varies continuously in $L^{p}$-topology for any $p \in[1, \infty)$.

Proposition 1. As $\alpha \rightarrow 2$, we have $P_{\alpha} \rightarrow P_{2}$ in $L\left(\mathrm{BV}_{0}, L^{p}\right)$-topology for any $p \in[1, \infty)$. More precisely, if $\alpha, \beta \in J \in \mathscr{A}_{n}$ then

$$
\left\|P_{\beta} \phi-P_{\alpha} \phi\right\|_{L^{p}} \leqq \text { const. }\left(\left|S_{n}^{\prime}\right|^{\omega_{0}}|\beta-\alpha|^{1 / p}+\left|S_{n}^{\prime}\right|^{-\omega_{1}}\right) \operatorname{Var}(\phi),
$$

where $\omega_{0}, \omega_{1}$ are positive constants.

Proof. We can write:

$$
P_{\alpha} \phi=\sum_{k=0}^{\infty} P_{\alpha, k}(\phi)
$$

where

$$
P_{\alpha, k} \phi=\left\{\begin{array}{l}
0 \text { outside of } T_{\alpha, k}\left(M_{\alpha, k}\right) \\
\left(\phi g_{\alpha, k}\right) \circ T_{\alpha, k}^{-1} \text { on } T_{\alpha, k}\left(M_{\alpha, k}\right) .
\end{array}\right.
$$

We have an obvious estimate

$$
\left\|P_{\alpha, k} \phi\right\|_{p} \leqq \sup g_{\alpha, k} \sup |\phi| \leqq \text { const. } \Lambda_{0}^{-k} \operatorname{Var}(\phi) .
$$

So the norms in $L\left(\mathrm{BV}_{0}, L^{p}\right)$-topology decay exponentially, uniformly in $\alpha \in A_{\infty}$. To show continuity we need to prove that for each $k$ "the difference" $P_{\alpha, k}-P_{\beta, k}$ is small in $L\left(\mathrm{BV}_{0}, L^{p}\right)$-topology, when $|\alpha-\beta|$ is small and $\alpha, \beta \in J \in \mathscr{A}_{n}$ for sufficiently large $n$.

Let $K$ be a maximal subsegment of $M_{\alpha, k}$ contained in the domain of the map $H_{\alpha \beta^{\prime}}=T_{\beta^{\prime}}^{-1} \circ T_{\alpha}$ and $M_{\beta^{\prime}, k}$ for all $\beta^{\prime} \in[\alpha, \beta]$. Since $\operatorname{Supp}\left(\left|P_{\alpha, k} \phi-P_{\beta, k} \phi\right|\right)=$ $T_{\alpha, k}\left(M_{\alpha, k}\right) \cup T_{\beta, k}\left(M_{\beta, k}\right)$ for $p \in[1,2)$ we can write:

$$
\begin{aligned}
\int_{I}\left|P_{\alpha, k} \phi-P_{\beta, k} \phi\right|^{p}= & \int_{S_{1}}\left|P_{\alpha, k} \phi\right|^{p}+\int_{S_{2}}\left|P_{\beta, k} \phi\right|^{p}+\int_{S_{3}}\left|P_{\alpha, k} \phi-P_{\beta, k} \phi\right|^{p} \\
& +\int_{T_{\alpha}(K)}\left|P_{\alpha, k} \phi-P_{\beta, k} \phi\right|^{p}
\end{aligned}
$$

where

$$
\begin{aligned}
& S_{1}=T_{\alpha, k}\left(M_{\alpha, k}\right) \backslash\left(T_{\beta, k}\left(M_{\beta, k}\right) \cup T_{\alpha}(K)\right), \\
& S_{2}=T_{\beta, k}\left(M_{\beta, k}\right) \backslash\left(T_{\alpha, k}\left(M_{\alpha, k}\right) \cup T_{\alpha}(K)\right), \quad \text { and } \\
& S_{3}=\left(T_{\alpha, k}\left(M_{\alpha, k}\right) \cap T_{\beta, k}\left(M_{\beta, k}\right)\right) \backslash T_{\alpha}(K) .
\end{aligned}
$$


Noting that $\left|P_{\alpha, k} \phi-P_{\beta, k} \phi\right|^{p} \leqq 2^{p-1}\left(\left|P_{\alpha, k} \phi\right|^{p}+\left|P_{\beta, k} \phi\right|^{p}\right)$ on $S_{3}$ and changing variables of integration, we get:

$$
\begin{aligned}
\int_{I}\left|P_{\alpha, k} \phi-P_{\beta, k} \phi\right|^{p} \leqq & 2^{p-1} \sup |\phi|^{p}\left(\left|M_{\alpha, k} \backslash K\right|+\left|M_{\beta, k} \backslash H_{\alpha \beta}(K)\right|\right) \\
& +\sup |\phi|^{p}\left(\left|M_{\alpha, k} \backslash H_{\beta \alpha}\left(M_{\beta, k}\right)\right|+\left|M_{\beta, k} \backslash H_{\alpha \beta}\left(M_{\alpha, k}\right)\right|\right) \\
& +\int_{T_{\alpha}(K)}\left|g_{\alpha, k} \phi-\left(g_{\beta, k} \phi\right) \circ H_{\alpha \beta}\right|^{p} \circ T_{\alpha}^{-1} \\
\leqq & 4 \sup |\phi|^{p}\left(\left|M_{\alpha, k} \backslash K\right|+\left|M_{\beta, k} \backslash H_{\alpha \beta}(K)\right|\right) \\
& +\int_{K}\left|\phi-\left(\phi \circ H_{\alpha \beta}\right) H_{\alpha \beta}^{\prime}\right|^{p}\left|g_{\alpha}\right|^{p-1} .
\end{aligned}
$$

The last term of this inequality can be estimated using the following

Lemma 1. Suppose that $H:[a, b] \rightarrow[c, d]$ is a homeomorphism and $\phi: \mathbb{R} \rightarrow \mathbb{R}$ is a function with bounded variation on a segment $U$ containing both $[a, b]$ and $[c, d]$. Then

$$
\int_{[a, b]}|\phi-\phi \circ H|^{p} \leqq \operatorname{dist}\left(\mathrm{id}, \mathrm{H}^{-1}\right) \operatorname{Var}_{U}^{p}(\phi) .
$$

Proof. Just in this proof let $\mu$ be a measure on $U$ such that $\phi(x)-\phi(y)=\int_{x}^{y} d \mu(t)$ for any $x, y \in U, x \leqq y$.

First, in view of the fact that $p \geqq 1$ we have the following obvious inequality:

$$
\begin{aligned}
|\phi(x)-\phi(H(x))|^{p} & \leqq|\phi(x)-\phi(H(x))| \cdot \operatorname{Var}_{U}^{p-1}(\phi) \\
& \leqq \operatorname{Var}_{U}^{p-1}(\phi) \int_{[x, H(x)]} d \mu .
\end{aligned}
$$

Integration of this inequality over $[a, b]$ (with respect to the Lebesgue measure and change of variables yield the following:

$$
\begin{aligned}
\int_{[a, b]}|\phi(x)-\phi(H(x))|^{p} d x & \leqq \operatorname{Var}_{U}^{p-1}(\phi) \int_{[a, b][x, H(x)]} \int_{H} d \mu d x \\
& \leqq \operatorname{Var}_{U}^{p-1}(\phi) \int_{U\left[t, H^{-1}(t)\right]} \int_{0} d x d \mu \\
& \leqq \operatorname{Var}_{U}^{p-1}(\phi) \int_{U} \operatorname{dist}\left(\mathrm{id}, H^{-1}\right) d \mu \\
& =\operatorname{dist}\left(\mathrm{id}, H^{-1}\right) \operatorname{Var}_{U}^{p}(\phi)
\end{aligned}
$$

This concludes the proof.

Therefore the last term of the inequality (51) admits the estimate:

$$
\begin{aligned}
\int_{K}\left|\phi-\left(\phi \circ H_{\alpha \beta}\right) H_{\alpha \beta}^{\prime}\right|^{p} \leqq & 2^{p-1} \operatorname{Var}^{p}(\phi) \operatorname{dist}\left(H_{\beta \alpha}, \mathrm{id}\right) \\
& +2^{p-1} \sup |\phi|^{p} \operatorname{dist}^{p}\left(H_{\alpha \beta}^{\prime}, 1\right)
\end{aligned}
$$

for $p \in[1,2)$. 
Note that the ends of $M_{\alpha, k}$ are Lipschitz with respect to $\alpha$ and let $L$ be the Lipschitz constant. Since $\frac{\partial}{\partial \alpha}\left(T_{\alpha, k}^{-1}\right)=-\delta\left(T_{\alpha, k}\right)$ the ends of $H_{\alpha \beta^{\prime}}(K)$ move with speed not exceeding $\sup _{\beta^{\prime} \in[\alpha, \beta]}\left|\delta\left(T_{\beta^{\prime}, k}\right)\right|$. It follows that $\operatorname{dist}\left(\partial K, \partial M_{\alpha, k}\right) \leqq$ $\left(L+\sup \left|\delta\left(T_{\beta^{\prime}, k}\right)\right|\right)|\beta-\alpha|$.

It is also easy to see that $\operatorname{dist}\left(H_{\alpha \beta}\right.$, id $) \leqq$ const. $\sup \left|\delta\left(T_{\beta^{\prime}, k}\right)\right||\beta-\alpha|$. Moreover,

$$
\left|\frac{\partial}{\partial \beta} \log \right| H_{\alpha \beta}^{\prime}||=\left(\left|T_{\beta}^{\prime}\right|\left|\Delta_{\beta x}\left(T_{\beta, k}\right)+\Delta_{x x}\left(T_{\beta, k}\right) \delta\left(T_{\beta, k}\right)\right|\right) \circ H_{\alpha \beta} .
$$

Combining these estimates with inequalities (3.18) in [11] we can easily complete the proof. Indeed,

$$
\left\|P_{\alpha, k} \phi-P_{\beta, k} \phi\right\|_{L^{p}} \leqq \text { const. }\left|S_{k}^{\prime}\right|^{\omega_{0}}|\alpha-\beta|^{1 / p} \operatorname{Var}(\phi),
$$

where $\omega_{0}>0$ is some constant. So, we can write:

$$
\left\|P_{\alpha}-P_{\beta}\right\|_{L\left(\mathrm{BV}_{0}, L^{p}\right)} \leqq \text { const. }|\alpha-\beta|^{1 / p} \sum_{k \leqq n}\left|S_{k}^{\prime}\right|^{\omega_{0}}+\text { const. } \sum_{k>n}\left|S_{k}^{\prime}\right|^{-\omega_{1}},
$$

where $\omega_{1}>0$ is determined by (50), and, using the fact that $\left|S_{k}^{\prime}\right| \leqq \Lambda_{0}^{-(n-k)}\left|S_{n}^{\prime}\right|$ for $k \leqq n$ and $\left|S_{k}^{\prime}\right| \geqq \Lambda_{0}^{k-n}\left|S_{n}^{\prime}\right|$ for $k>n$, bring the last expression to the form in the statement of the proposition.

Theorem 6. If $\alpha$ is a Misiurewicz point then there exists $\omega_{2}>0$ such that for any $\phi \in B V_{0}$ and $\beta \in A_{\infty}$ we have:

$$
\left\|P_{\alpha} \phi-P_{\beta} \phi\right\|_{L^{p}} \leqq \text { const. }|\alpha-\beta|^{\omega_{2}} \operatorname{Var}(\phi) .
$$

Proof. From construction of $A_{n}$ in [11] it follows that for a Misiurewicz point $\alpha$ there is $J \in A_{n}$ which contains $\alpha$ and $\beta$ for any $n \leqq$ const. $\log \left(1 /|\alpha-\beta|^{\omega_{3}}\right)$. We can also prove that $\left|S_{n}^{\prime}\right| \geqq \lambda^{n}$, where $\lambda>1$ (for Misiurewicz points the sequence $s_{n}$ grows linearly). Therefore, we can pick $n$ in such a fashion that $\left|S_{n}^{\prime}\right| \leqq$ const. $|\alpha-\beta|^{-\omega_{3}} \leqq\left|S_{n+1}^{\prime}\right|$. We immediately notice that $\left|S_{n}^{\prime}\right| \geqq$ const. $|\alpha-\beta|^{-\omega_{3}}$. Plugging these inequalities into the inequality from the last proposition, one can easily show the desired inequality.

We will need the following well-known fact:

Lemma 2. The norm of $P$ in $L^{p}$-topology does not exceed $\left\|P^{1}\right\|_{\infty}^{1-1 / p}$.

Proof. The proof is based on Jensen's inequality and we leave it to the reader.

Corollary 1. If $\sup _{n}\left\|P^{n} 1\right\|_{\infty}<\infty$ then the operators $P^{n}$ have uniformly bounded norms in $L^{p}$ topology for $p \in[1, \infty]$.

Let $\rho_{\alpha}$ be the density of $\bar{\nu}_{\alpha}$ (the unique a.c.i.m. for $T_{\alpha}$ ). We set out now to prove the following

Theorem 7. The function $A_{\infty} \ni \alpha \mapsto \rho_{\alpha} \in L^{p}(m)$ is continuous at Misiurewicz points. Moreover, for every $p \in[1, \infty)$ there is a constant $\omega_{4}>0$ such that if $\alpha \in A_{\infty}$ is a Misiurewicz point then:

$$
\left\|\rho_{\alpha}-\rho_{\beta}\right\|_{L^{p}} \leqq \text { const. }|\alpha-\beta|^{\omega_{4}}
$$

for all $\beta \in A_{\infty}$. 
Proof. This proof incorporates the same ideas as a corresponding proof in [10]. Let $h=\rho_{\alpha}-\rho_{\beta}$, so that $\rho_{\alpha}=\rho_{\beta}+h$. Rewriting the equation $P_{\alpha}\left(\rho_{\beta}+h\right)=\rho_{\beta}+h$, we obtain:

$$
\left(I-P_{\alpha}\right) h=u \stackrel{\text { def }}{=}\left(P_{\alpha}-P_{\beta}\right) \rho_{\beta}
$$

One can easily see that $u \in \mathrm{BV}_{0}$ and is uniformly bounded (i.e. $\operatorname{Var}(u)$ is uniformly bounded with respect to $\alpha \in A_{\infty}$ ). This follows from the uniform basic inequality (see Sect. 2).

Therefore, we can write the von Neumann series expressing $h$ in terms of $u$ :

$$
h=\sum_{n=0}^{\infty} P_{\alpha}^{n} u .
$$

We can estimat the $L^{p}$-norm of $h$ by splitting the series into a finite part and the tail:

$$
\begin{aligned}
\|h\|_{L^{p}} & \leqq \sum_{n=0}^{N}\left\|P_{\alpha}^{n}\right\|_{L^{p}}+\sum_{n=N+1}^{\infty}\left\|P_{\alpha}^{n}\right\|_{L^{p}} \\
& \leqq \text { const. } N\|u\|_{L^{p}}+\sum_{n=N+1}^{\infty} \text { const. } \lambda^{n}\|u\|_{\mathrm{BV}_{\mathrm{o}}} \\
& \leqq \text { const. } N\|u\|_{L^{p}}+\text { const. } \lambda^{N} .
\end{aligned}
$$

From the definition and the previous theorem we known that $\|u\|_{L^{p}} \leqq$ const. $|\alpha-\beta|^{\omega_{2}}$. Using an optimal choice of $N$, we arrive at the desired inequality.

\section{Continuity of the Invariant Density II}

Let $\hat{\rho}_{\alpha}$ be the density of the invariant measure $v_{\alpha}\left(f_{\alpha}\right.$-invariant). It is not difficult to show that this density admits the following representation in terms of the invariant density $\rho_{\alpha}$, which has been proved to be continuous at Misiurewicz points:

$$
\hat{\rho}_{\alpha}=\sum_{n=0}^{\infty} \sum_{j=0}^{s_{n}-1} \rho_{\alpha, n, j},
$$

where

$$
\rho_{\alpha, n, j}=\left\{\begin{array}{l}
\frac{\rho_{\alpha}}{\left(f^{j}\right)^{\prime}} \circ\left(f^{j} \mid M_{\alpha, n}\right)^{-1} \text { on } f^{j}\left(M_{\alpha, n}\right) \\
0 \text { otherwise }
\end{array} .\right.
$$

The first result we intend to prove in this section was announced in [11] and is analogous to a result proved in [1].

Proposition 2. The series (62) converges in $L^{p}$-topology uniformly with respect to $\alpha \in A_{\infty}$ for all $p \in[1,2)$. 
Proof. We may assume that $j \geqq 1$; the case $j=0$ is easy. By changing coordinates we can easily show that

$$
\int_{f^{j}\left(M_{\alpha, n}\right)}\left|\frac{\rho_{\alpha}}{\left(f^{j}\right)^{\prime}}\right|^{p} \circ\left(f^{j} \mid M_{\alpha, n}\right)^{-1} d m=\int_{M_{\alpha, n}} \frac{\left|\rho_{\alpha}\right|^{p}}{\left|\left(f^{j}\right)^{\prime}\right|^{p-1}} d m .
$$

Let $l \in \mathbb{Z}$ be such that $s_{l}>j \geqq s_{l-1}$. Let $i=s_{l}-j$. From our definitions it follows that $S_{l}=f^{i} \circ f^{j-1}, l \leqq n$ and $i \leqq s_{k_{l}}$. Therefore the chain rule yields:

$$
\inf _{V_{n}}\left|\left(f^{j-1}\right)^{\prime}\right| \geqq \frac{\inf \left|S_{l}^{\prime}\right|}{\left(\max \left|f^{\prime}\right|\right)^{i}} \geqq \frac{\inf \left|S_{l}^{\prime}\right|}{4^{\beta}} \geqq\left(\frac{\Lambda_{0}}{4^{\beta}}\right)^{l} .
$$

In particular, $\inf _{V^{n}}\left|\left(f^{j-1}\right)^{\prime}\right| \geqq$ const. (we recall that $\beta$ was a small constant, so that $\left.\Lambda_{0}>4^{\beta}\right)$. Therefore, on $M_{\alpha, n}$ we have $\left|\left(f^{j}\right)^{\prime}\right| \geqq$ const. $|x|$. This implies that

$$
\int_{M_{\alpha, n}} \frac{\left|\rho_{\alpha}\right|^{p}}{\left|\left(f^{j}\right)^{\prime}\right|^{p-1}} d m \leqq \text { const. } \int_{M_{\alpha, n}} \frac{d m}{|x|^{p-1}} \leqq \text { const. }\left|I_{n}\right|^{2-p} .
$$

The last number converges to 0 exponentially uniformly in $\alpha \in A_{\infty}$, as was shown in [11]. Therefore, the infinite series (62) can be majorized in $L^{p}$-topology by the exponentially convergent series:

$$
\sum_{n=0}^{\infty} S_{n}\left|I_{n}\right|^{2 / p-1}
$$

(We recall that $s_{n} \leqq \beta n^{2} / 2$ ).

Our next objective is to show that $\hat{\rho}_{\alpha}$ is continuous at Misiurewicz points. It is clear that in order to prove this it suffices to show that each term of the series (62) is continuous in $L^{p}$-topology for $p \in[1,2)$.

For this part of the proof it will be convenient to fix $n$ and $j$ and introduce the following notation: $T_{\alpha}=f^{j} \mid M_{\alpha, n}, \mathscr{D}_{\alpha}=M_{\alpha, n}$ for domain of $T_{\alpha}, \mathscr{R}_{\alpha}=T_{\alpha}\left(\mathscr{D}_{\alpha}\right)$, and $H_{\alpha \beta}=T_{\beta}^{-1} \circ T_{\alpha}$. Let us also denote $\rho_{\alpha, n, j}$ by $\sigma_{\alpha}$. In this simplified notation we can write:

$$
\sigma_{\alpha}=\left\{\begin{array}{l}
\frac{\rho_{\alpha}}{\left|T_{\alpha}^{\prime}\right|} \circ T_{\alpha}^{-1} \text { on } \mathscr{R}_{\alpha} \\
0 \text { otherwise }
\end{array} .\right.
$$

Our goal is to estimate $\left\|\sigma_{\alpha}-\sigma_{\beta}\right\|_{L^{p}}$ for two close parameters $\alpha$ and $\beta$ and $p \in[1,2)$. This estimate is somewhat similar to the estimate of the previous section.

Let $K$ be the maximal segment contained in all the segments $\mathscr{D}_{\alpha} \cap \mathscr{D}_{\beta^{\prime}}$ $\cap H_{\alpha \beta^{\prime}}\left(\mathscr{D}_{\alpha}\right)$ for every $\beta^{\prime} \in[\alpha, \beta]$. We can write

$$
\int_{I}\left|\sigma_{\alpha}-\sigma_{\beta}\right|^{p} d m=\int_{\left(\mathscr{R}_{\alpha} \cup \mathscr{R}_{\beta}\right) \backslash T_{\alpha}(K)}\left|\sigma_{\alpha}-\sigma_{\beta}\right|^{p} d m+\int_{T_{\alpha}(K)}\left|\sigma_{\alpha}-\sigma_{\beta}\right|^{p} d m .
$$

We have:

$$
\begin{aligned}
\int_{\left(\mathscr{R}_{\alpha} \cup \mathscr{R}_{\beta}\right) \backslash T_{\alpha}(K)}\left|\sigma_{\alpha}-\sigma_{\beta}\right|^{p} d m & =\int_{\left.\mathscr{R}_{\alpha} \backslash\left(\mathscr{R}_{\beta} \cup T_{\alpha}\right) \backslash(K)\right)}\left|\sigma_{\alpha}\right|^{p} d m \\
+ & \int_{\mathscr{R}_{\beta} \backslash\left(\mathscr{R}_{\alpha} \cup T_{\alpha}(K)\right)}\left|\sigma_{\beta}\right|^{p} d m+\int_{\left(\mathscr{R}_{\alpha} \cap \mathscr{R}_{\beta}\right) \backslash T_{\alpha}(K)}\left|\sigma_{\alpha}-\sigma_{\beta}\right|^{p}
\end{aligned}
$$




$$
\begin{aligned}
& \leqq \sup \left|\rho_{\alpha}\right|^{p} \int_{\mathscr{D}_{\alpha} \backslash K} \frac{1}{\left|T_{\alpha}^{\prime}\right|^{p-1}} d m+\sup \left|\rho_{\beta}\right|^{p} \int_{\mathscr{D}_{\beta} \backslash K} \frac{1}{\left|T_{\beta}\right|^{p-1}} d m \\
&+2^{p-1}\left(\int_{\mathscr{D}_{\alpha} \backslash K} \frac{\left|\rho_{\alpha}\right|^{p}}{\left|T_{\alpha}^{\prime}\right|^{p-1}} d m+\int_{\mathscr{D}_{\beta} \backslash K} \frac{\left|\rho_{\beta}\right|^{p}}{\left|T_{\alpha}^{\prime}\right|^{p-1}} d m\right) \\
& \leqq \text { const. } 2^{p}\left(\sup \left|\rho_{\alpha}\right|^{p} \int_{\mathscr{D}_{\alpha} \backslash K} \frac{1}{|x|^{p-1}} d m+\sup \left|\rho_{\beta}\right|^{p} \int_{\mathscr{D}_{\beta} \backslash K} \frac{1}{|x|^{p-1}} d m\right) \\
& \leqq \text { const. }\left(\sup \left|\rho_{\alpha}\right|^{p}\left|\mathscr{D}_{\alpha} \backslash K\right|^{2-p}+\sup \left|\rho_{\beta}\right|^{p}\left|\mathscr{D}_{\beta} \backslash K\right|^{2-p}\right) .
\end{aligned}
$$

Meanwhile,

$$
\begin{aligned}
\int_{T_{\alpha}(K)}\left|\sigma_{\alpha}-\sigma_{\beta}\right|^{p}= & \int_{K} \frac{\left|\rho_{\alpha}-\rho_{\beta} \circ H_{\alpha \beta} H_{\alpha \beta}^{\prime}\right|^{p}}{\left|T_{\alpha}^{\prime}\right|^{p-1}} d m \\
\leqq & 3^{p-1}\left(\int_{K} \frac{\left|\rho_{\alpha}-\rho_{\beta}\right|^{p}}{\left|T_{\alpha}^{\prime}\right|^{p-1}} d m+\int_{K} \frac{\left|\rho_{\beta}-\rho_{\beta} \circ H_{\alpha \beta}\right|^{p}}{\left|T_{\alpha}^{\prime}\right|^{p-1}}\right. \\
& \left.+\sup \left|\rho_{\beta}\right|^{p} \int_{K} \frac{\left|1-H_{\alpha \beta}^{\prime}\right|^{p}}{\left|T_{\alpha}^{\prime}\right|^{p-1}} d m\right) \\
\leqq & \operatorname{const.} 3^{p-1}\left(\int_{K} \frac{\left|\rho_{\alpha}-\rho_{\beta}\right|^{p}}{|x|^{p-1}} d m+\int_{K} \frac{\left|\rho_{\beta}-\rho_{\beta} \circ H_{\alpha \beta}\right|^{p}}{|x|^{p-1}}\right. \\
& \left.+\sup \left|\rho_{\beta}\right|^{p} \int_{K} \frac{\left|1-H_{\alpha \beta}^{\prime}\right|^{p}}{|x|^{p-1}} d m\right) .
\end{aligned}
$$

The terms of the last formula can be estimated as follows. Let us pick positive numbers $q$ and $r$ such that $1 / q+1 / r=1$ and $(p-1) r<1$. Then by Hölder's inequality

$$
\begin{gathered}
\int_{K}\left|\rho_{\alpha}-\rho_{\beta}\right|^{p}|x|^{1-p} d m \leqq\left(\int_{K}\left|\rho_{\alpha}-\rho_{\beta}\right|^{p q}\right)^{1 / q}\left(\int_{K}|x|^{(1-p) r}\right)^{1 / r}, \\
\int_{K}\left|\rho_{\alpha}-\rho_{\beta} \circ H_{\alpha \beta}\right|^{p}|x|^{1-p} d m \leqq\left(\int_{K}\left|\rho_{\alpha}-\rho_{\beta} \circ H_{\alpha \beta}\right|^{p q} d m\right)^{1 / q}\left(\int_{K}|x|^{(1-p) r}\right)^{1 / r}, \\
\int_{K}\left|1-H_{\alpha \beta}^{\prime}\right|^{p}|x|^{1-p} d m \leqq\left(\int_{K}\left|1-H_{\alpha \beta}^{\prime}\right|^{p q} d m\right)^{1 / q}\left(\int_{K}|x|^{(1-p) r}\right)^{1 / r},
\end{gathered}
$$

and

$$
\left(\int_{K}|x|^{(1-p) r}\right)^{1 / r} \leqq \frac{|K|^{1 / r-(p-1)}}{(1-r(p-1))^{1 / r}} .
$$

We notice that if $p$ is close to 2 then $r$ has to be close to 1 and $q$ has to be very large. Therefore, we need the $L^{p}$ estimates of the previous section with large $p$. 
Lemma 3.' The following inequalities are true:

$$
\begin{aligned}
& \int_{K}\left|\rho_{\beta}-\rho_{\beta}{ }^{\circ} H_{\alpha \beta}\right|^{p} d m \leqq \operatorname{dist}\left(\mathrm{id}, H_{\beta \alpha}\right) \|_{\mathrm{BV}}^{p} \\
& \int_{K} \frac{\left|1-H_{\alpha \beta}^{\prime}\right|^{p}}{\left|T_{\alpha}^{\prime}\right|^{p-1}} \leqq \sup _{\beta^{\prime} \in[\alpha, \beta]}\left|T^{\prime}\right|^{2-p}\left(\Delta_{\beta^{\prime} x}(T)+\Delta_{x x}(T)|\delta(T)|\right)^{p}|\alpha-\beta|^{p} \\
& \operatorname{dist}\left(\mathrm{id}, H_{\beta \alpha}\right) \leqq \sup |\delta(T)||\alpha-\beta| .
\end{aligned}
$$

It is clear now that we need estimates for $\delta(T), \Delta_{\alpha x}(T)$, and $\Delta_{x x}(T)$.

Lemma 4. For any $p \in[1, \infty)$ there is $\omega>0$ such that

$$
\delta(T), \Delta_{\alpha x}(T), \Delta_{x x}(T) \leqq \text { const. }\left|S_{n}^{\prime}\right|^{\omega} .
$$

Proof. The proof follows from the possibility to represent $f^{j} \mid M_{\alpha, n}$ for $j \leqq s_{n}(j \geqq 1)$ as a composition:

$$
f^{j} \mid M_{\alpha, n}=T_{l_{s}, \alpha} \circ \cdots \circ T_{l_{2}, \alpha} \circ S_{l_{1}, \alpha} \circ\left(f \mid M_{\alpha, n}\right),
$$

where the first $s$ expanding maps form a $\beta$-homogeneous sequence in the terminology of [11]. The proof of this lemma is then completely similar to the proof of Lemma 3.1 and Theorem 3.4 of [11].

Combining all the estimates of this section we obtain

Theorem 8. For every $p \in[1,2)$ there are positive constants $\omega_{4}, \omega_{5}$ such that for any Misiurewicz point $\alpha$ and $\beta \in A_{\infty}$ we have

$$
\begin{gathered}
\left\|\rho_{\alpha, n, j}-\rho_{\beta, n, j}\right\|_{L^{p}} \leqq \text { const. }|\alpha-\beta|^{1 / p}\left|S_{n}^{\prime}\right|^{\omega_{4}}, \\
\left\|\hat{\rho}_{\alpha}-\hat{\rho}_{\beta}\right\|_{L^{p}} \leqq \text { const. }|\alpha-\beta|^{\omega_{5}} .
\end{gathered}
$$

Remark 2. Our estimates allow us to prove continuity (but not Hölder continuity) of $\hat{\rho}_{\alpha}$ at the points $\alpha \in \tilde{A}_{\infty} \subset A_{\infty}$. Construction of $\tilde{A}_{\infty}$ is very similar to that of $A_{\infty}$; at the $n^{\text {th }}$ stage, instead of deleting only $\psi_{n}^{-1}\left(\left[-\lambda^{-\sqrt{n}}, \lambda^{-\sqrt{n}}\right]\right)$ we also delete arbitrarily small neighborhoods (e.g. $\sim \lambda^{-\sqrt{n}}$ ) of the endpoints of $I_{\alpha, k}$. It is easy to see that $m\left(\tilde{A}_{\infty}\right)>0$, and continuity follows from the fact that $|\alpha-\beta|<\varepsilon$ implies that $\alpha, \beta \in J \in \mathscr{A}_{n}$ for some $n=n(\varepsilon)$, which tends to $\infty$ when $\varepsilon \rightarrow 0$. As in the case of $A_{\infty}$, Misiurewicz points are dense and they are Lebesgue density points of $\tilde{A}_{\infty}$.

Remark 3. The proofs presented here apply to $A_{\infty}$ built near the special Misiurewicz point $\alpha=2$. A complete proof for other Misiurewicz points requires additional constructions.

\section{Continuity of the Lyapunov Exponent}

Let us recall the Rokhlin formula for the Lyapunov exponent,

$$
\text { Lyapunov exponent of } f_{\alpha}=h_{v_{\alpha}}=\int_{I} \log \left|f_{\alpha}^{\prime}\right| d v_{\alpha}
$$


converges uniformly. As a consequence of our estimates of the continuity of the invariant density the Lyapunov exponent is continuous at the Misiurewicz points $\alpha$ close to 2 .

Theorem 9. The Lyapunov exponent of $f_{\alpha}$, as $\alpha \rightarrow 2$ and $\alpha \in A_{\infty}$, tends to $\log 2$. Moreover, there is a constant $\omega>0$ such that if $\Gamma_{\alpha}$ is the Lyapunov exponent computed for the parameter $\alpha \in A_{\infty}$ and $\beta$ is a Misiurewicz point sufficiently close to 2 then

$$
\left|\Gamma_{\alpha}-\Gamma_{\beta}\right| \leqq \text { const. }|\alpha-\beta|^{\omega} .
$$

Proof. The formula (81) yields an explicit expression for the Lyapunov exponent of $f_{\alpha}$ in terms of the invariant measure.

A simple calculation shows that

$$
\Gamma_{\alpha}-\Gamma_{\beta}=\int_{I} \log |x|\left(\hat{\rho}_{\alpha}-\hat{\rho}_{\beta}\right) d m+\log \frac{\alpha}{\beta} .
$$

By the Hölder inequality for $p=3 / 2$ we obtain the following inequality:

$$
\left|\int_{I} \log \right| x\left|\left(\hat{\rho}_{\alpha}-\hat{\rho}_{\beta}\right) d m(x)\right| \leqq\left(\int_{I}(\log |x|)^{3} d x\right)^{1 / 3}\left\|\hat{\rho}_{\alpha}-\hat{\rho}_{\beta}\right\|_{L^{3 / 2}} .
$$

As a result, if $\beta$ is a Misiurewicz point near 2 then we can apply the results of Sect. 6 to obtain the last statement of our theorem.

\section{Appendix A: The Measure of the Central Segment}

The objective of this appendix is to estimate $v(C)$, where $C=(-\gamma, \gamma)$.

Lemma 5. The density of $f_{*}^{j}\left(\bar{v} \mid M_{\alpha, n}\right)$, which we called $\rho_{\alpha, n, j}$, for $j \in\left\{1,2, \ldots, s_{n}-1\right\}$ admits the estimate

$$
\left|\rho_{\alpha, n, j}(x)\right| \leqq \text { const. } \frac{\left|x-f^{j-1}(1)\right|^{-1 / 2}}{\left|\left(f^{j-1}\right)^{\prime}(1)\right|^{1 / 2}} .
$$

Proof. As we have shown already, the map $f^{j-1} \mid M_{\alpha, n}$ has bounded distortion. Moreover, the density of the measure $f_{*}\left(\bar{v} \mid M_{\alpha, n}\right)$ is bounded by const. $|x-1|^{-1 / 2}$. From the formula (62) for $\hat{\rho}_{\alpha}$ we know that the density $\rho_{\alpha, n, j}$ can be bounded by

$$
\frac{\text { const. }\left|1-f^{-(j-1)}(x)\right|^{-1 / 2}}{\left|\left(f^{j-1}\right)^{\prime}(1)\right|} \text {. }
$$

Also, because of the bounded distortion,

$$
\left|1-f^{-(j-1)}(x)\right| \approx\left|x-f^{j-1}(1)\right|\left|\left(f^{j-1}\right)^{\prime}(1)\right|^{-1} .
$$

This yields the desired estimate.

Proposition 3. Let $C=(-\gamma, \gamma)$. Then:

$$
|v(C)-\bar{v}(C)| \leqq \text { const. } \gamma^{\log \Lambda_{0} / \beta},
$$

where $\beta$ has the same meaning as in (78). 
Proof. From the definitions and the previous lemma it follows that

$$
\begin{aligned}
v(C)-\bar{v}(C) & =\sum_{n=0}^{\infty} \sum_{j=1}^{s_{n}-1} \int_{C \cap f^{j}\left(M_{\alpha, n}\right)} \rho_{\alpha, n, j} d m \\
& \leqq \text { const. } \sum_{n=0}^{\infty} \sum_{j=1}^{s_{n}-1} \frac{\left|C \cap f^{j}\left(M_{\alpha, n}\right)\right|^{1 / 2}}{\left|\left(f^{j-1}\right)^{\prime}(1)\right|^{1 / 2}} .
\end{aligned}
$$

It is easy to see from the definitions of [11] that for $j \leqq s_{n}-1$ we have $f^{j}\left(M_{\alpha, n}\right) \cap I_{l}=\emptyset$, where $l=\max _{m \leqq n} k(m)$. Also we have

$$
\left|I_{l}\right|=\text { const. }\left|S_{l}^{\prime}(1)\right|^{-\tau} \geqq 4^{-\tau \operatorname{deg} S_{l}} \geqq 4^{-\tau \beta n} .
$$

Therefore, for $n<(1 / 2 \tau \beta) \log (1 / \gamma)$ we have $f^{j}\left(M_{\alpha, n}\right) \cap C=\emptyset$. Hence, for sufficiently small $\gamma$ we have the following estimate:

$$
|v(C)-\bar{v}(C)| \leqq \text { const. } \sum_{n \geqq(1 / 2 \tau \beta) \log (1 / \gamma)} \sum_{j=1}^{s_{n}-1} \frac{\left|f^{j}\left(I_{\alpha, n}\right)\right|^{1 / 2}}{\left|\left(f^{j-1}\right)^{\prime}(1)\right|^{1 / 2}} .
$$

We have an easy estimate $\left|f^{j}\left(I_{\alpha, n}\right)\right| \leqq$ const. $\left|\left(f^{j-1}\right)^{\prime}(1)\right|\left|I_{\alpha, n}\right|^{2}$. Combining this with previous inequality, we obtain:

$$
\begin{aligned}
|v(C)-\bar{v}(C)| & \leqq \text { const. } \sum_{n \geqq(1 / 2 \tau \beta) \log (1 / \gamma)} \beta n^{2}\left|I_{\alpha, n}\right| \\
& \leqq \text { const. } \Lambda_{0}^{-(1 / \beta) \log (1 / \gamma)} \\
& =\text { const. } \gamma^{\frac{\log \Lambda_{0}}{\beta}} .
\end{aligned}
$$

This concludes the proof.

\section{Appendix B: Positivity of the Lyapunov Exponent}

There is a very short self-contained proof of the fact that for the parameters $\alpha \in A_{\infty}$ the Lyapunov exponent is positive for $f_{\alpha}(x)=1-\alpha x^{2}$. One has to assume that the measure $v$ is obtained from $\bar{v}$, as described above.

The properties below which follow from the construction will be useful:

1. $s_{k} \leqq$ const. $k^{2}(k \geqq 1)$.

2. $\left|I_{k}\right| \leqq$ const. $\lambda^{k}$ for some $\lambda \in(0,1)$.

3. $\left|T^{\prime}\right| \geqq \Lambda>1$.

We define functions $\phi(x)=\log \left|T^{\prime}(x)\right|$ and $n(x)=s_{k}$, as $\quad x \in I_{k} \backslash I_{k+1}$ $(k=0,1,2, \ldots)$. To compute the Lyapunov exponent for $f_{\alpha}$ we consider:

$$
\frac{\phi(x)+\phi(T x)+\cdots+\phi\left(T^{l-1} x\right)}{n(x)+n(T x)+\cdots+n\left(T^{l-1} x\right)}
$$

This number is

$$
\frac{1}{N} \log \left|\left(f_{\alpha}^{N}\right)^{\prime}(x)\right|
$$

where $N$ is the denominator of Eq. (92). Hence taking $l \rightarrow \infty$ in (92) yields the Lyapunov exponent of $f_{\alpha}$ for $v$-almost every $x$. We can rewrite (92) as follows: 


$$
\frac{\frac{1}{l} \sum_{i=0}^{l-1} \phi\left(T^{i} x\right)}{\frac{1}{l} \sum_{i=0}^{l-1} n\left(T^{i} x\right)} .
$$

The last limit exists for $\bar{v}$-almost every $x$ by the Ergodic Theorem, applied to $(T, \bar{v})$. The limit is positive, provided that the denominator does not blow up to $\infty$. This is true if $\int_{I} n(x) d \bar{v}(x)<\infty$. But using Properties $1-3$ we can see that

$$
\int_{I} n(x) d \bar{v}(x) \leqq \text { const. }\left(1+\sum_{k=1}^{\infty} k^{2} \lambda^{k}\right)<\infty .
$$

More precisely, Lyapunov exponent $\geqq \log \Lambda / \int n(x) d \bar{v}(x)$. We would like to emphasize that this inequality holds for the Lyapunov exponent computed at $\bar{v}$-almost every point. It is also true for almost every point with respect to $v$, since the support of $v$ is the union of $f_{\alpha}$-forward images of the support of $\bar{v}$.

From the previous sections it follows that the measure $v$ is unique (up to a constant) and that the support of $v$ is the segment $\left[f_{\alpha}(1), 1\right]$. Each point of $I$ or its image belongs to this interval, so the Lyapunov exponent exists for almost every point in $I$. Because $v$ is unique, it is ergodic. This implies that the Lyapunov exponent is a well-defined constant, depending on $\alpha \in A_{\infty}$ only.

Acknowledgements. We would like to express our gratitude to T. Spencer for suggesting the problem of continuity of entropy as a research topic. We would also like to thank the Institute for Advanced Study, Courant Institute of Mathematical Sciences, and the National Science Foundation for funding this research.

\section{References}

1. Benedicks, M., Carleson, L.: On iterations of 1- $a x^{2}$ on ( - 1, 1). Ann. Math. (2) 122 (1), 1-25 (1985)

2. Bowen, R.: Equilibrium States and the Ergodic Theory of Anosov Diffeomorphisms. Lecture Notes in Mathematics, vol. 470, Berlin, Heidelberg, New York: Springer-Verlag 1975

3. Hofbauer, F., Keller, G.: Ergodic properties of invariant measures for piecewise monotonic transformations. Math. Z. 180, 119-140 (1982)

4. Krzyżewski, K., Szlenk, W.: On invariant measures for expanding differentiable mappings. Studia Math. 23, 83-92 (1969)

5. Krzyżewski, K.: On connection between expanding mappings and Markov chains. Bull. Acad. Pol. Sci. Ser. Math., vol. 19, 291-293 (1971)

6. Keller, G.: On the Rate of Convergence to Equilibrium in One-Dimensional Systems. Commun. Math. Phys. 96, 181-193 (1984)

7. Ledrappier, F.: Some properties of absolutely continuous measures on an interval. Ergodic Theory Dyn. Syst. 1, 77-93 (1981)

8. Jakobson, M.V.: Absolutely continuous invariant measures for one-parameter families of one-dimensional maps. Commun. Math. Phys. 81, 39-88 (1981)

9. Rychlik, M.: Bounded variation and invariant measures. Studia Math. t. 76, 69-80 (1983)

10. Rychlik, M.: Regularity of the Metric Entropy for Expanding Maps. Preprint, IAS, 1988

11. Rychlik, M.: Another proof of Jakobson's theorem and related results. Engrdic Theory Dyn. Syst. 8, 93-109 (1988)

12. Young, L.-S.: Decay of Correlations for Certain Quadratic Maps. Preprint, UCLA, 1991

13. Ziemian, K.: Almost sure invariance principle for some maps of an interval. Engrdic Theory Dyn. Syst. 5, 625-640 (1985) 